7-15-2020

\title{
Are more people adopting COVID-19 prevention measures?
}

UNICEF

Population Council Institute

Follow this and additional works at: https://knowledgecommons.popcouncil.org/departments_sbsr-pgy

Part of the Demography, Population, and Ecology Commons, International Public Health Commons, and the Public Health Education and Promotion Commons How does access to this work benefit you? Let us know!

\section{Recommended Citation}

UNICEF and Population Council Institute. 2020. "Are more people adopting COVID-19 prevention measures?" COVID-19 Research Results Brief \#2. New Delhi: UNICEF and Population Council Institute.

This Brief is brought to you for free and open access by the Population Council. 
ARE ME The adoption of preventive measures has increased over time. Even so, just a

ARE MORE PEOPLE ADOPTING COVID-19 little less than two-fifths of males and one-quarter of females reported that they and their family members were currently practicing all four standard preventative measures.

\section{BACKGROUND}

In India, the number of COVID-19 positive cases has increased substantially in recent weeks. With the easing of lockdown, albeit for justifiable reasons, there has been an increase in movement of people from red zones to green zones and vice-versa. It is important that people continue to adhere to the government guidelines on preventive measures.

Drawing on data from two rounds (Phase 1 and the earlier research) of COVID-19 knowledge, attitudes and practices (KAP) telephone surveys conducted in Bihar, this research brief sheds light on the socio-economic differentials and changes over time in the adoption of COVID-19 preventive measures.

\section{METHODS}

The sample households for the KAP surveys were drawn from an existing state-representative cohort study of adolescents and young adults UDAYA (www.projectudaya.in) study, in Bihar. However, it is noted that respondents who participated in the COVID-19 KAP surveys were better educated, wealthier and less likely to have belonged to disadvantaged castes and tribes (SC/ST) compared with those who did not. There were no differences in terms of rural-urban or religious distributions.

- In the earlier round of the survey (April 3-12, 2020, 175 men and 385 women were interviewed and in the Phase 1 round of the current study (May 13-22, 2020), 242 men and 552 women were interviewed, including 111 men and 246 women who participated in both rounds of the survey (see the table below for a profile of participants in the two rounds).

\section{PROFILE OF STUDY PARTICIPANTS}

\section{Characteristics}

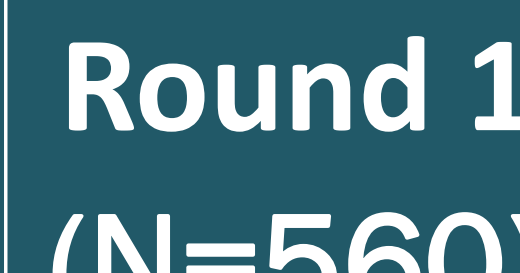

Round 2 $(\mathrm{N}=560) \quad(\mathrm{N}=794)$

Mean age

Mean years of education

$\%$ Female

$\%$ Hindu

Caste (\%)

$\mathrm{SC} / \mathrm{ST}$

OBC

General

Wealth index (quintiles as of

UDAYA 2015-16 survey)

Bottom two quintiles (poor)

Medium

Top two quintiles (rich)

\section{0}

$10.0 \quad 11.1$

$68.7 \quad 69.5$

89.5

85.5

$20.5 \quad 16.4$

$\begin{array}{ll}20.5 & 16.4 \\ 61.1 & 63.7\end{array}$
$18.4 \quad 19.9$
RISK PERCEPTION AND PREVENTIVE

\section{PRACTICES}

A larger proportion of participants who perceived

a moderate to high risk of COVID-19 in their neighbourhood reported that they \& their family members practiced all four standard preventive measures than those who perceived no/low risk.

\section{ACTION TAKEN BY SELF AND HOUSEHOLD MEMBERS ${ }^{1}$}

Male

Stayed at home (not stepping outside unless urgent)

Washed hands/used hand sanitizer

more frequently

Started using masks

Kept a distance of at least 1-2 meters

All of the above

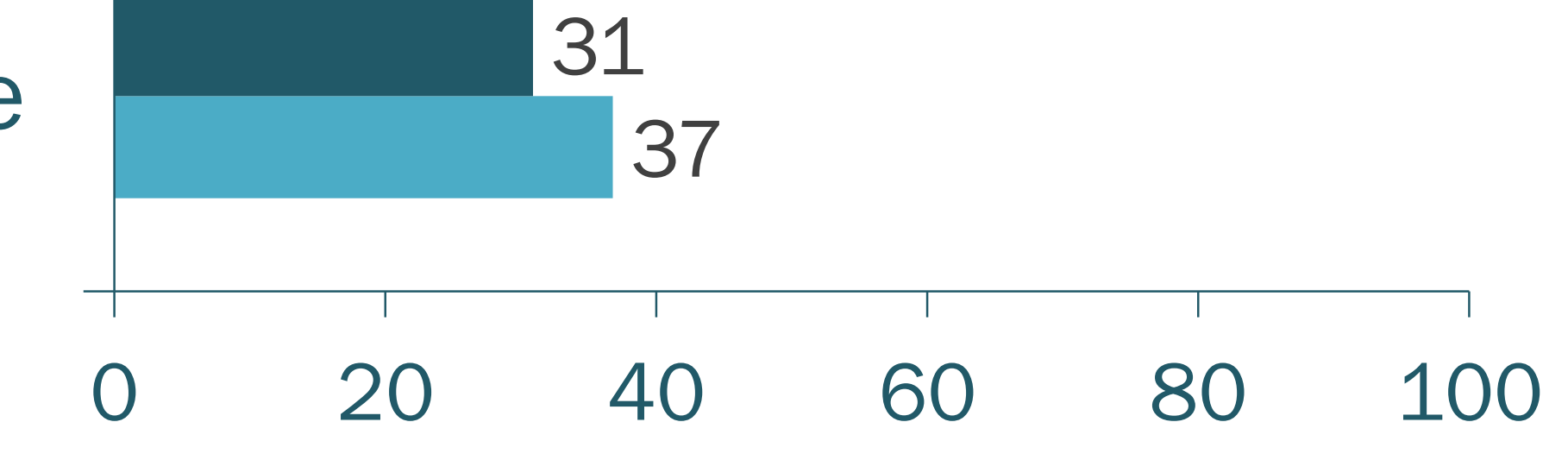

- Round 1 Round 2
Female
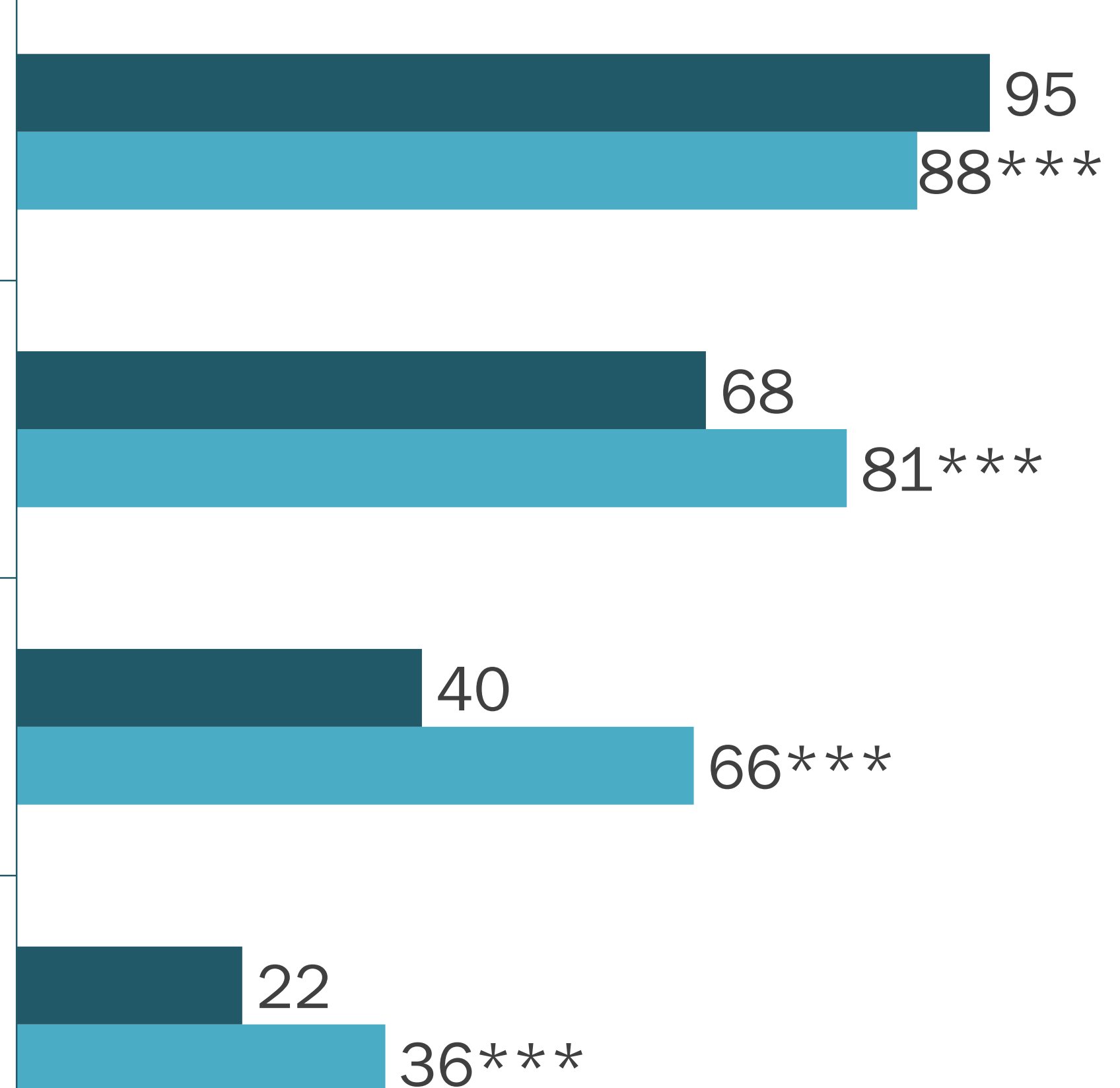
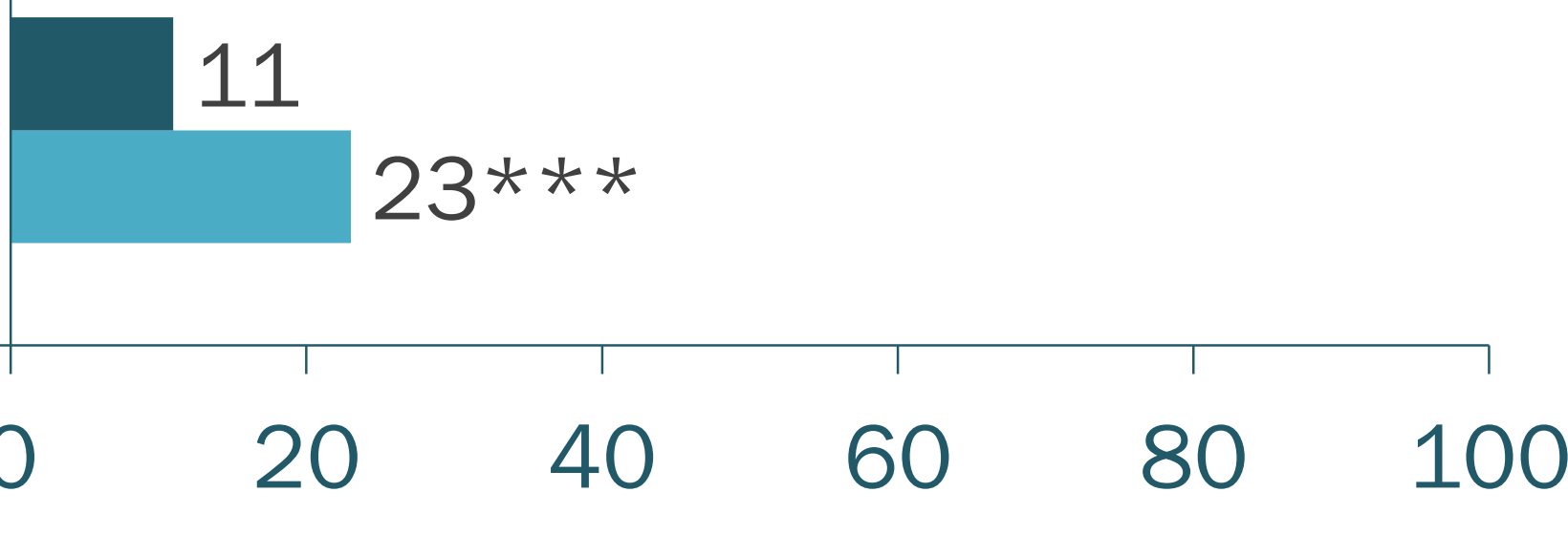

- Round 1 Round 2
Note: ${ }^{1}$ Based on answers given spontaneously in response to a single question about the preventive practices adopted by respondents and their household members; percentages less than 10\% are not shown in Figure. Significance level: $* p \leq 0.05, * * p \leq 0.01, * \star * p \leq 0.001$

- The proportion of males and females who reported that they \& their family members were staying at home unless urgent declined over time. At the same time, those who reported that they $\&$ their family members were practising social distancing of 1-2 meters increased.

The proportion of females who reported frequent hand washing and using masks increased over time.

While there was no statistically significant difference in the proportion of males who reported that they \& their family members were practising all four common measuresstaying at home, frequent hand washing, using masks and keeping social distance of 1-2 meters-between the two rounds of survey (31\% at round 1 and $37 \%$ at round 2), more female respondents reported so in round 2 than in round 1 (23\% and 11\%).

- More males than females reported that they \& their family members were practising all four common preventive measures (31\% vs. $11 \%$ in round 1 and $37 \%$ vs. $23 \%$ in round 2).

\section{COVID-19 PREVENTION PRACTICES² BY SOCIO-ECONOMIC CHARACTERISTICS}

\section{Age in completed} years $(\%)$

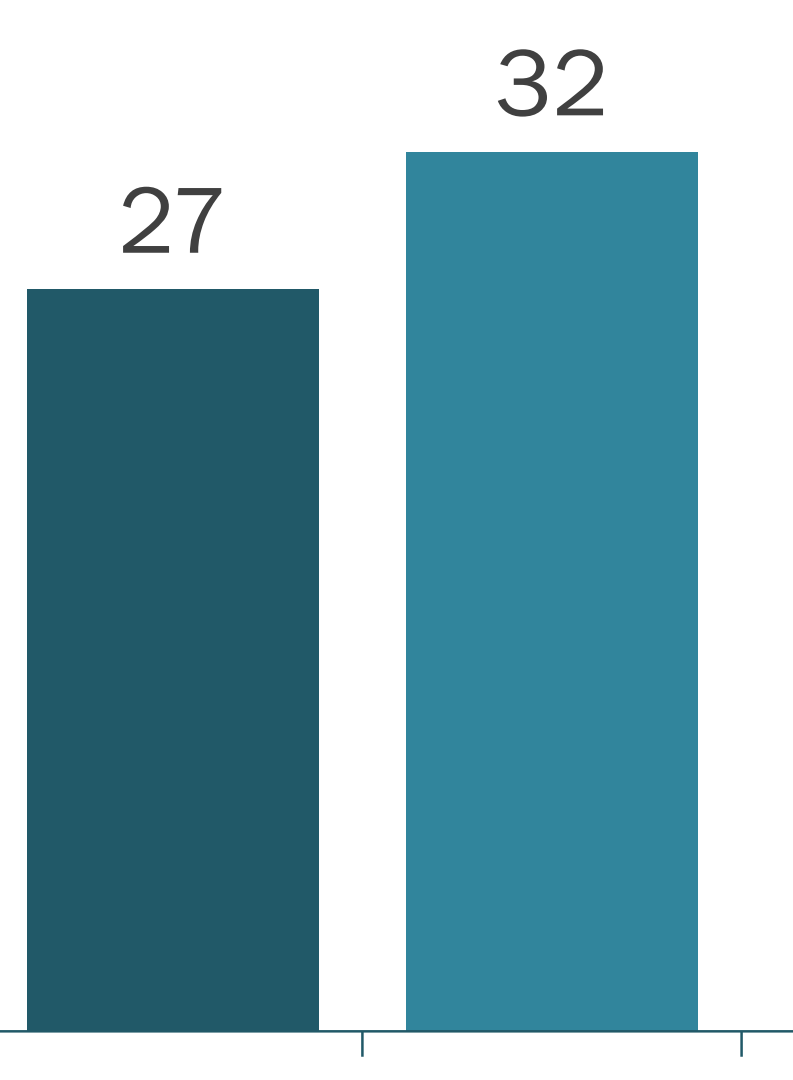

18-24 25 or
Years of education

(\%)

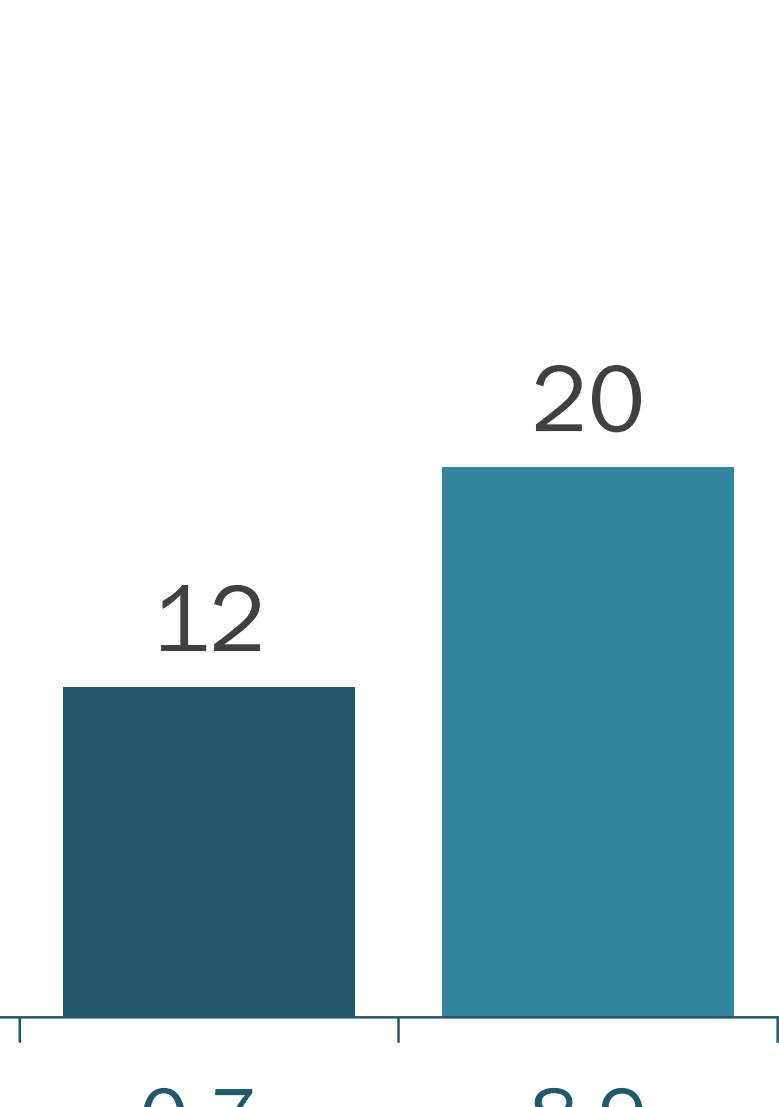

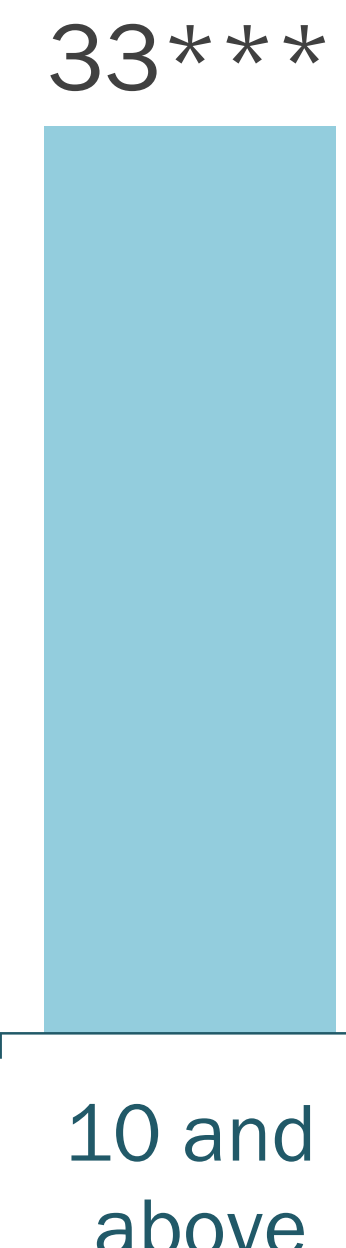

Caste $(\%)$

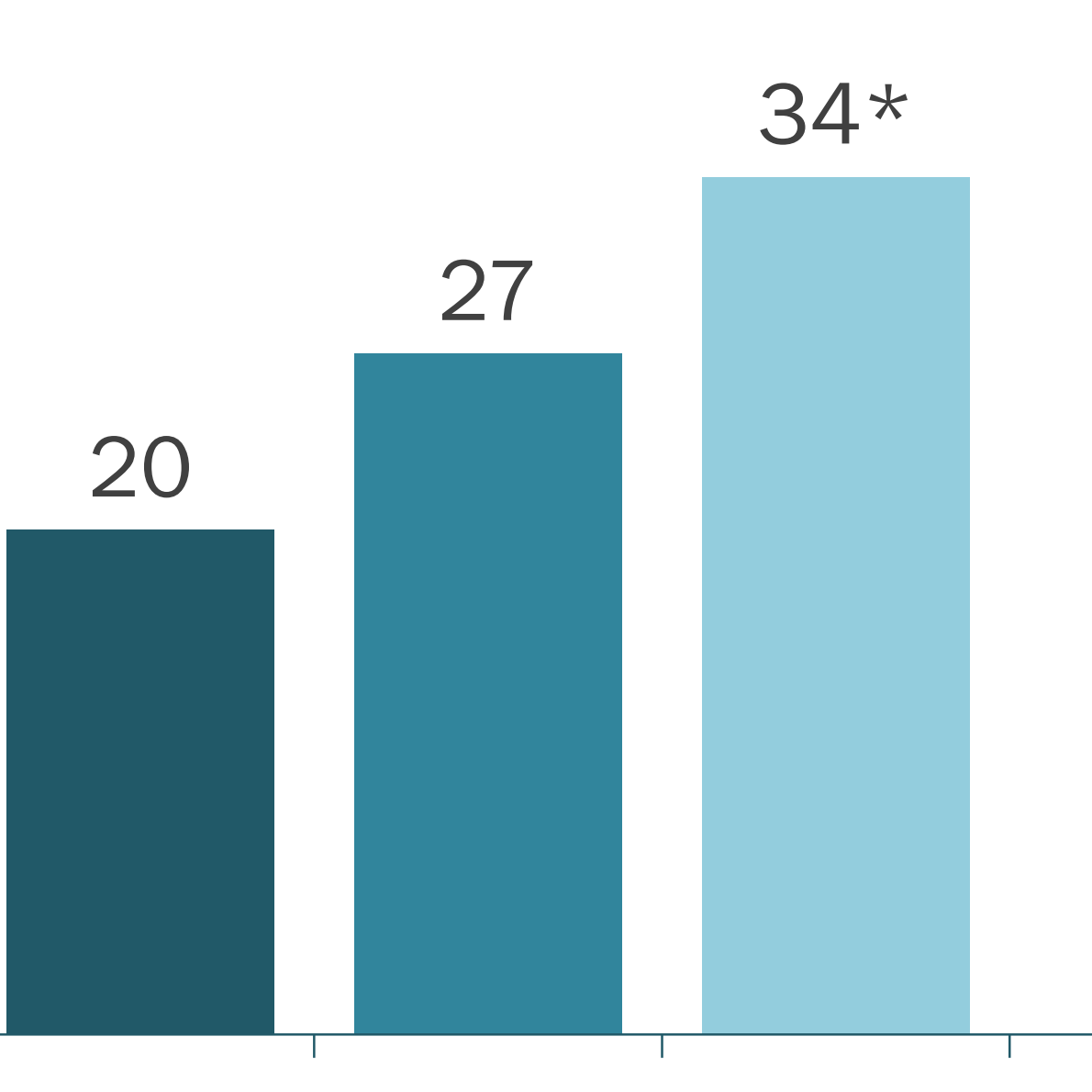

SC/ST
Wealth index (quintiles)

Note: $2 \%$ who practised all four common preventive measures; Significance level: $* p \leq 0.05$ and $* \star \star p \leq 0.001$

Reported practice of all four common preventive measures increased with education, household economic status and caste hierarchy.

\section{LIMITATIONS}

$>$ Findings cannot be generalised to the overall situation in Bihar given the selectivity bias in the sample as mentioned in the Method section.

- Given efforts by many stakeholders to raise awareness about preventive measures, the chances of respondents giving socially desirable responses cannot be ruled out.

$>$ Findings were based on self-reports and not based on observations.

\section{CONTACT INFORMATION}

This work was jointly undertaken by Population Council Institute and UNICEF/Bihar.

For more information, contact fzavier@popcouncil.org; kgsanthya@popcouncil.org; msinha@unicef.org 